\title{
EDUCACIÓN INTERCULTURAL. ¿TRABAJAR CON LOS DIFERENTES O LAS DIFERENCIAS?
}

Juan Bello Domínguez

\section{Resumen}

En el marco de la Política Educativa formulada en el contexto Internacional por los Organismos Multilaterales (Jomtiem, 1990; Salamanca, 1994; Dakar, 2000), se enfatizó la obligación moral y política, de atender también a los grupos sociales que expresen y manifiesten características sociales, culturales, económicas, políticas y físicas diferentes, que exige una sociedad democrática.

Las necesidades socioculturales y educativas de los colectivos planteadas en estos foros, fueron postuladas a partir del diálogo entre la inclusión y la interculturalidad, para "garantizar" el acceso a todos los servicios educativos de los que dispone la sociedad, "asegurandoles" los recursos para socializarse, crecer sin perder su identidad e incorporarse a los procesos socioeconómicos.

Las persistentes brechas sociales, culturales, económicas y educativas, evidenciaron el número de personas que viven en la pobreza y, la distribución desigual de la riqueza; dichas brechas se convirtieron en serias limitantes, además de demorar los procesos de democratización de las sociedades. Sin embargo, más que ser una cuestión marginal relativa a la integración de los educandos en el sistema educativo regular, el debate se centra en, cómo transformar los sistemas educativos y los entornos de aprendizaje, para dar respuesta a las diferencias de los educandos y no a la atención de los diferentes.

Palabras Clave: Educación, Intercultural, Inclusión, Diversidad, Marginación. 


\section{Abstract}

In the context of the Educative Politic formulated in the international scope by the Multilateral Organisms (Jomtiem, 1990; Salamanca, 1994; Dakar, 2000), it's been emphasized the moral and political obligation of attend also the social groups that express and manifest social, cultural, economic, politic, and physical different characteristics, that a democratic society requires.

The sociocultural and educative necessities of the groups raised in this forums, were postulated from the dialog between the inclusion and the interculturality for guarantee the access to all the educative services available to society, "assuring" the resources for socialize, grow without losing their identity and been incorporated to socioeconomic processes.

The persistent social, cultural, economic, and educative gaps, showed the number of people that lives in poverty and, the unequal distribution of the wealth; those gaps has been transformed in serious deficiencies, moreover of delay the democratization of the societies processes. However more than been a relative marginal issue to the integration of the students in the regular educative system, the debate focuses in how to transform the educative systems and the knowledge environments for giving an answer to the differences of the students and not to the attention of the different.

Key Words: Education, Intercultural, Inclusion, Diversity, Marginalization

\section{INTRODUCCIÓN}

La urgencia de comprender la diversidad, la inclusión y la interculturalidad en la sociedad y las escuelas, aumenta el debate sobre la existencia de desacuerdos, tensiones y disputas, así como al afianzamiento de las desigualdades e iniquidades que aparecen en ellas. Lo importante es comprender e identificar los recursos y actitudes que permitan afrontar los significados más profundos y sus alcances en la sociedad y su diversidad.

Resulta importante llamar la atención sobre la diversidad, la inclusión y la interculturalidad, porque representa un signo en el proceso de democratización de las sociedades contemporáneas, porque a partir de ésta, se promoverá el reemplazo de la visión homogenea insertados por los Estados nacionales durante el siglo pasado. Estas tensiones entre la diversidad-homogeneidad se superarán al generar cambios importantes en torno a los procesos sociales y culturales. Las sociedades orientadas a partir del multiculturalismo, evidenciaron la falta de claridad de sus políticas para atender lo diverso. (Kymlicka, 1996 y 2003)

\section{EXCLUSIÓN, UN ACERCAMIENTO CONCEPTUAL}

Las concepciones de la democracia en los inicios de su historia moderna, estuvieron basadas en la esperanza de un futuro mejor. Las sociedades promovieron su futuro en la utopía de alcanzar espacios de convivencia, comunicación y desarrollo socioeconómico y cultural, por ello, en nombre de los valores universales de la democracia occidental, se ha tratado de "modernizar a viejas o extrañas" civilizaciones.

En el contexto histórico y hasta mediados del siglo XIX, se conceptualizó la sociedad en términos políticos: paz y guerra, orden y desorden. (Touraine, 2005) Eran las categorías que estructuraban la visión y la práctica. Luego, las representaciones y organización se expresaron en términos económicosociales, un modelo en el que los conceptos eran capital, trabajo, huelgas y mercado. $Y$ todo eso se ha ido abajo, no estamos ya en ese paradigma, ya que el nuevo paradigma con el que se da cuenta de todas estas preocupaciones conceptuales, es el cultural. (Touraine, 2005)

stablecer el paradigma de la diversidad, la inclusión y el diálogo intercultural en el contexto sociocultural, implica generar escenarios que lo favorezcan frente a identidades, que se presentan en las demarcaciones culturales y tienden a su hibridación; identidades de larga data que mantienen cierto rango de flexibilidad y en su recreación, les permite conjugar las permanencias con culturas diferentes y sus relaciones.

Se trata desde el punto de vista de Touraine (1998 y 1997), de la transformación del individuo en Sujeto: primero, a través del reconocimiento del otro, también como Sujeto, que gobierna la comunicación intercultural, y en un segundo momento, de la reconstrucción de la vida personal y colectiva, fundada sobre la idea de que el sujeto personal y la comunicación de los sujetos entre sí, necesitan protecciones institucionales reemplazando la democracia como participación en la voluntad general, por la idea de instituciones al servicio de la libertad del Sujeto y del diálogo entre éstos. 


\section{EDUCACIÓN EN TORNO A LA INTERCULTURALIDAD}

Si hablamos de inclusión social o escolar, la expresión misma nos remite a la "exclusión" en el marco de las instituciones. En el contexto histórico de la sociedad y de las instituciones educativas, los testimonios refieren la búsqueda de la homogenización como una forma de inclusión legal pero no legitimada, ya que a partir del desconocimiento de la formas diversas de expresión social, cultural, lingüística, de género o física, se ejerce la exclusión formal.

Hablar de inclusión en una sociedad excluyente, nos remite nuevamente al compromiso impuesto a la escuela, de legitimar y defenderen un marco de desigualdades. La democracia planteada en términos formales, no es suficiente. No basta el reconocimiento formal de las instituciones para fortalecer y consolidar la inclusión escolar. (Bello, 2010)

Durante los últimos años, se desarrollaron políticas "específicas" para atender las necesidades sociales y educativas de la población (UNESCO, 1990, 1994, 2000). Las características de estas políticas, mostraron una amplia diversidad en sus formas concretas en el diseño y su instrumentación, cuyo propósito era dar cuenta de las características de los diseños y acciones llevadas a cabo por la política social y educativa, en el contexto de la diversidad y las características socioeducativas y lingüistas de la población, las cuales manifiestan grados de marginación, exclusión y desigualdad.

Nos cuestionamos ¿quiénes somos?, ¿cuál es la matriz cultural que nos da sentido?, ¿qué nos identifica y nos diferencia? Los espacios sociales, políticos, culturales y educativos en los que se expresa la diferenciación, la identidad, la pluralidad y de resistencia frente a los otros, a lo otro, lo que no es nuestro, fija nuestros límites, nuestro ser y nuestro modo de ser diferentes, nuestra personalidad propia y nuestra expresión particular, donde la política, la economía, la educación y la sociedad han dado origen a las ideas de alteridad, soberanía y autonomía.

a conformación de identidades y organizaciones en el marco de la globalización, exige la idea de que el propósito del desarrollo no es solamente el crecimiento económico, sino que el desarrollo debiera estar estrechamente relacionado con los niveles de bienestar. (UNESCO, 1997) Se hace indispensable un escenario que pueda generar el diálogo cultural y educativo, crear vínculos entre sujetos, manifestar diferencias, solidaridades, resistencias, discrepancias; pero sobre todo, que sea un marco en donde la interculturalidad sea el principio organizador que propicie el contacto entre culturas, para la reelaboración constante de las mismas y la negociación de las diferencias en el marco de la construcción de las identidades.

Los problemas de identidad y conformación del imaginario colectivo, conllevan a dirimir sobre las posibilidades y los límites del papel de la cultura y la educación en las relaciones interpersonales, los movimientos sociales y su condición de marginación y exclusión. En el discurso, se reconocen los derechos a ser diferentes, a la libre determinación y a la autonomía de las comunidades escolares, pero no existe voluntad política para llevar a cabo las transformaciones en la sociedad y la escuela, para la alteridad, la otredad y la diversidad.

Se vive en sociedades diferenciadas, en contraposición con la idea de homogeneidad expresada en los imaginarios de la igualdad ciudadana construidos historicamente en torno a los Estados nacionales, por ello, es preciso articular la búsqueda de las identidades individuales y grupales, con la búsqueda del diálogo democrático de la sociedad. Los retos de la conformación de la ciudadanía, llevan a enriquecer la identidad de la cultura y la educación en su pluralidad y expresiones, y junto con ello, a proyectar las culturas locales, populares y comunitarias en todos los espacios, como parte de la estrategia de las políticas que se adopten, no sólo desde el punto de vista de generar igualdad de oportunidades y compensación de diferencias, sino como un compromiso que conduzca hacia un desarrollo equilibrado, bajo la cohesión (que no homogeneización) de grupos y la participación activa, que compense en equidad de oportunidades.

Es necesario reconstruir los vínculos y las relaciones, ante la complejidad de las formas de hacer cultura o el contacto entre imaginarios más allá de las oposiciones entre lo global, regional o local. Para abordar la cuestión de la identidad, no es suficiente señalar la oposición entre lo local, regional y lo global, hay que recuperar los espacios de intermediación social, cultural, educativa y política, y tener en cuenta que no todos los ámbitos y sectores sociales, son tocados por la globalización con la misma intensidad. (Garcia, 1999)

No basta con la formulación de políticas basadas en el respeto mutuo y la libertad cultural, como lo traza el multiculturalismo; se hace necesario el ejercicio de la libertad cultural y educativa para la construcción del dialogo y la convivencia. El momento histórico, exige el ejercicio y la participación democrática que dé respuesta a las expectativas de la diversidad de pueblos y culturas que forman parte de la sociedad. En este sentido, la cultura y la educación se convierten en propósito y base social del desarrollo, como la 
realización de la existencia del ser humano en forma integral, como la posibilidad de que la cultura pueda enriquecerse y desarrollarse como fuente de progreso y creatividad; como una posibilidad, no sólo de construir el desarrollo, sino la vida misma en todas sus dimensiones.

El desarrollo social no tiene por qué estar reñido con las culturas y la educación, tendría que ser nutrido por éstos, pensándolos como vetas de creatividad, lo cual implica que el término desarrollo debiera ser dinámico, cambiante, flexible a cada espacio y situación.

\section{¿TRABAJAR CON LOS DIFERENTES O LAS DIFERENCIAS?}

La educación se encuentra ante una grave crisis de identidad, los procesos cambiantes y dinámicos de la sociedad han desplazado a la escuela a un ámbito de falta de credibilidad y desconfianza en donde los profesores, alumnos y directivos entraron en tensiones en torno a sus expectativas. No estamos hablando de un problema de eficacia, que se podría resolver mediante una mejora técnica, sino de legitimidad.

La escuela, en su época más brillante, surge en un contexto de certezas (fabricación de la ciudadanía, integración social y laboral de los ciudadanos, socialización metódica). Sin embargo, también con promesas no cumplidas (movilidad social e igualdad de oportunidades), que fueron malogradas y la abocaron a su actual incertidumbre. No solo fue incapaz de promover la igualdad de oportunidades, sino que, se develo también en toda su crudeza, su papel crucial en la reproducción de las desigualdades sociales y culturales que prometía combatir.

Se mostró ineficiente para hacer frente a las condiciones de incertidumbre que genera la diversidad, flexibilidad, complejidad e inequidad. (Bauman, 2011)

Los "expertos educativos" se mostraron siempre listos, con base a los indicadores "malos" y "déficits" o "promesas incumplidas", para iniciar una nueva reforma. Se emplearon las estadísticas para demostrar crisis sociales $y$, de este modo, justificar los remedios educativos. Se orientaron las Políticas Educativas para la atención de los diferentes, con la "convicción" que habría que integrarlos a procesos sociales, económicos y productivos. Erradicar las diferencias para lograr la "igualdad" en bien de todos los miembros de la sociedad.

Habría que tener cuidado con las posiciones reformistas, ya que la escuela cambia el mensaje que ingresa, más de lo que el mensaje nuevo puede cambiar a la escuela. Justamente porque la mayoría de reformas tratan de introducir cambios en la estructura existente y quedan -al final-, absorbidas o acomodadas por ellas.

La sociedad y las escuelas, caminaron por senderos de crisis e inequidad en la atención a la diversidad, en los que les ha sido difícil salir y sobre todo, de encontrar paradigmas que permitan expresar nuevas y diferentes representaciones sociales para trabajar en y para la diversidad. Postularon cambios de paradigma (al menos de manera explícita) y siguieron apegadas a modelos homogenizantes tras una impresionante retórica curricular.

El sistema escolar se burocratizo y ahora es juzgado críticamente como "culpable" del "fracaso de loables intentos reformadores", de distorsionar el proceso de enseñanza para la "igualdad", de paralizar el desarrollo de las instituciones escolares para lograr la "integración" o la "inclusión". Predominante en nuestros sistemas educativos, se han favorecido políticas educativas impregnadas de uniformismo, en sus diferentes subsistemas para atender a "los discapacitados", o a "los indios".

Las escuelas constituyen, hoy día, contextos caracterizados por la diversidad de sus alumnos, reflejo, a su vez, de la sociedad cada vez más diversa. Los estudiantes son hoy más heterogéneo en su composición cultural, étnica, lingüística, de clase, de género, de capacidad. La presencia de grupos con sus correspondientes perspectivas culturales y religiosas, de alumnos con alguna discapacidad, o que provienen de familias con graves problemas sociales $y$ económicos etc. constituye una realidad que no es irrelevante a lo que acontece en las escuelas. (González, 2008)

Se concibe una nueva escuela que no sólo dé respuestas, sino que también enseñe a hacerse preguntas, que mire al pasado y también integre el futuro, no excluir a nadie de la formación a la que tienen derecho por razones de justicia y democracia. También se reclama esa mirada amplia sobre la diversidad, la diferencia y los fenómenos de exclusión por parte de la justicia social y la educación. Su planteamiento gira en tomo a la importancia de hacer de las escuelas instituciones más equitativas y justas.

Cuando el funcionamiento en la escuela se articula sobre una lógica individualista, se corre el riesgo de ofrecer un currículo y enseñanza que, a la postre no será sino una mera agregación de actuaciones dispersas y escasamente coordinadas. El planteamiento de una escuela que no excluya, conlleva cambios profundos en la sociedad y la institución. Tales cambios 
incluyen los supuestos, principios, creencias y valores vehiculados por la acción pedagógica, los lenguajes utilizados así como las normas no escritas.

La atención a la diversidad cobra sentido desde el convencimiento en la teoría y en la práctica, de que la diversidad es un valor en sí misma y que enriquece todos los procesos educativos y vivenciales; el diálogo con otros significados, formas de vida y personalidades concretas, es necesario para profundizar en la relación y participación democrática propias de la sociedad actual. Educar en la diversidad, implica la superación de la cultura oficial, para abordar la cultura a partir de las culturas particulares que aportan los educandos. Esto supone, la exigencia para el docente de adoptar una óptica multicultural e intercultural que se encamine hacia la valoración de las culturas silenciadas en el currículum, valorando otras formas de conocer y de vivir, permitiendo que expresen sus ideas y experiencias aquellas personas que no han sido escuchadas habitualmente en la toma de decisiones que les afectan.

Educar en la diversidad implica que, se asuma la igualdad como un valor, que permite la mejora de la sociedad mediante la mejora de sus componentes, facilitándoles la elección de sus propios caminos para su desarrollo y bienestar. El ser diferente no marca las líneas respecto a sus oportunidades, incluyendo la igualdad de condiciones en que se recibe educación mediante la oferta curricular.

Se realizan consideraciones en torno a las características de inequidad y exclusión que privan en el sistema educativo nacional, volviendo los ojos hacia sus costos y alcances. No es posible seguir pensando a la educación como una totalidad homogénea, debido al ritmo y desarrollo diferentes de las dimensiones sociales, a la tendencia específica que lleva a someter a éstas, a la lógica nacional de las leyes en las que subyace el principio de organización social.

Es imprescindible revertir el principio educativo que tiene el propósito de uniformar y ejercer su dominio para imponer proyectos únicos, en donde las dimensiones educativas, culturales, sociales y políticas, son ajenas a los grupos sociales con características socioculturales y educativas diferentes. Ha sido importante cuestionar el proyecto educativo como el espacio en el que se eximen las diferencias y se supere la contradicción inherente entre el proyecto nacional y los diferentes grupos de la sociedad mexicana.

Gestar una política educativa de la diferencia en la que se reconozca la diversidad y las demandas que genera para dar paso al pluralismo -- es el reto del proyecto educativo --, desde el punto de vista de la participación de diversos grupos sociales, con prácticas socioculturales diferentes en las que se contienen sus principios y conciencia del grupo. Para el logro del proyecto educativo, se hace indispensable un escenario plural de respeto a las diferencias y a la diversidad, pero sobre todo, de participación que conduzca a la discusión, al diálogo y a los consensos.

La tensión que provoca el encuentro alternativo entre la diversidad, no se superará sin generar cambios importantes en torno a los procesos educativos, culturales y sociales. Las experiencias constituidas a partir de la interculturalidad, han planteado un papel importante en el debate sobre las políticas educativas y sobre el destino de las instituciones. (Dietz, 2011)

Demandemos que se asuma una filosofía política, social y educativa en contra de la exclusión; una apuesta contra la discriminación. El aprendizaje de la alteridad y de la diversidad se ha convertido en algo prioritario.

Aspiramos a un modelo educativo alternativo intercultural, que responda a las expectativas de la población, desarrollemos procesos educativos y culturales que las comunidades reconozcan como legítimos y les asegure, la construcción de su experiencia social e histórica.

En las instituciones educativas no es suficiente ver solamente la institucionalización del espacio curricular en lo formal, pues no solo en los propósitos declarados o en las razones de ser, encontramos respuesta a la problemática sociocultural y educativa; habría que considerar como prioritarios aquellos innumerables rasgos, propósitos creencias y valores transmitidos tácitamente a través de las relaciones comunitarias y fundamentalmente las experiencias que caracterizan la vida escolar.

Destacar la forma en que se relaciona la educación con la sociedad y ponderar su contribución, alude a conceptualizar la educación intercultural en el marco de las sociedades democráticas. Las persistentes brechas sociales incrementaron la proporción de personas que viven en la desigualdad y la inequidad, dichas brechas se convierten en limitantes de toda sociedad moderna y demoran los procesos democráticos y educativos.

La situación en que se encuentran los sistemas educativos, no sólo es producto de sus propias contradicciones, sino también por las generadas y reproducidas por la sociedad. Así, tener un buen sistema educativo no es suficiente para acabar con la desigualdad, ya que ésta, tiene que ver con la inequitativa distribución de la riqueza, y por lo tanto, del poder y de las oportunidades. 
La tensión que provocó el encuentro entre las tendencias de la diversidadhomogeneidad, no se superará sin generar cambios importantes en torno a los procesos sociales. Las sociedades y los sistemas educativos constituidos a partir de las concepciones del multiculturalismo, han evidenciado la falta de claridad de sus políticas sobre lo diverso, por ello los sectores culturales y étnicamente diferenciados han conquistado un papel importante, como actores del debate sobre las políticas públicas y sobre el destino de las instituciones.

a lucha por el reconocimiento de los derechos colectivos, se desprendió de la especificidad cultural de los diversos pueblos, etnias, regiones, y comunidades, constituyéndose en el eje del desarrollo sociocultural, mostrando un avance notable. La estructuración de las demandas educativas, como alternativas posibles y su nivel de crecimiento en torno a la organización de sus instituciones, permitió a los diversos grupos y a sus actores sociales insertarse en los escenarios locales, regionales y nacionales.

Ponemos en tela de juicio el proyecto de la Política Pública, donde a través de su discurso sistemático y demagógico, deja entrever muchas deficiencias a la hora de que este proyecto se lleva acabo y la falta de proyectos particulares, que se adecuen a las necesidades de las culturas de cada región geográfica, lo que hay, son proyectos generales que se muestran pretenciosos y que no se sustentan en el desarrollo social, económico y cultural de las regiones y comunidades.

Pensar las instituciones educativas en el marco de la diversidad, inclusión e interculturalidad, exige la reflexión para la elaboración de políticas culturales y educativas, que contribuyan a enriquecer el ámbito democrático, entre los procesos económico, social, cultural, educativo y lingüístico.

Establecer el diálogo intercultural para generar mayores posibilidades al mismo; construir escenarios que lo favorezcan y considerar el desafío frente a identidades que atraviesan las demarcaciones culturales; fortalecer las identidades de larga data que mantienen cierto rango de flexibilidad en su recreación para permitir conjugar las permanencias con culturas diferentes, con las que coexisten y se relacionan. Ver el mundo como un escenario de diálogo, en donde la multiplicidad de culturas no se subordinan, sino se complementan.

Dentro de este marco de relaciones socioculturales y pedagógicas, se construiyen nuevos centros de concertación, negociación y rupturas. Es así, como existen semejanzas, diferencias y discordancias, construyendo nuevos ámbitos de interacción, donde el sentido de las prácticas colectivas y educativas se produce, reproduce y confronta, a partir de nuevos esquemas de negociación y disputa económica, social y cultural

Los cambios educativos implican una profunda redefinición del papel de los actores sociales y una mayor incorporación de los referentes culturales. La identidad se construye en la relación entre lo individual, educativo y social, dentro de un marco histórico y simbólico; las transformaciones en lo referente a la identidad se insertan en prácticas cotidianas a través de la familia, el trabajo, las condiciones objetivas de vida o a través de la identificación con imaginarios sociales.

Asistimos a un fenómeno globalizador en el contexto socioeducativo y sus principios, son aleatorios al sistema educativo y a los valores culturales de grupos, pueblos y naciones; su repercusión más sensible esta en el imaginario social, donde subyace la idea de vigencia al derecho de los pueblos a ejercer soberanía, en relación con el control sobre su patrimonio cultural: bienes y recursos materiales, territorios, lengua, formas de organización social, conocimientos, creencias, vías de desarrollo y las lealtades necesarias que garantizan que la comunidad se mantenga y se reproduzca.

La apertura a la alteridad es un requisito de la interculturalidad. Quien no es capaz de reconocerle a cada cultura sus valores y pretensiones de universalidad, no está preparado para asumir la multiculturalidad. Por ello, el multiculturalismo sólo tiene sentido como la combinación, en un territorio dado, con una relativa unidad social, de una pluralidad de culturas, que mantienen permanentes intercambios y comunicaciones entre actores, que utilizan diferentes sentidos de la vida. La coexistencia o simple convivencia de culturas no expresa necesariamente la condición multicultural. 


\section{FUENTES DE INFORMACIÓN}

Bauman, Zygmunt. (2011) Daños Colaterales. Desigualdades sociales en la era global, , México: Ed. FCE

Bello Domínguez, Juan y Vivian G. Fernández Dávila Urquidi (Coord). (2012) Derechos Humanos de los Pueblos Indigenas en América Latina. Perspectivas y Retos., México: Facultad de Estudios Superiores Aragón, UNAM

Bello Domínguez, Juan. (2010). "La Educación Intercultural en el Contexto de la Diversidad y la Inclusión" en Bello Domínguez, Juan y Elisa Velásquez (Comp), La Educación Interculturalen el Contexto de la Diversidad y la Inclusión, México: Castellanos Editores

Bello Domínguez, Juan y Rosa María Salgado (Coord), (2010) Espacios interculturales en la América Latina de/ Siglo XXI, México: Ed. Universidad Pedagógica Nacional

Bello Domínguez, Juan. (2007) Educación y Pueblos Excluidos. México: Ed. Senado de la República, LX Legislatura, Facultad de Estudios Superiores Aragón, UNAM y Miguel Ángel Porrua.

Bello Domínguez, Juan. (2011) "La Pobreza y Marginación de los Pueblos Indios en México frente al Desarrollo Sustentable" en María Luisa Quintero y Carlos Fonseca (Coord) Dimensiones Económicas, Sociales e Institucionales del Desarrollo Sustentable. México: Ed. Cámara de Diputados y Miguel Ángel Porrua.

Bello Domínguez, Juan. (2010) "De la Educación Bilingüe Bicultural, a las Universidades Interculturales en México" en Eduardo Andrés Sandoval Forero, Ernesto Guerra García y Ricardo Contreras Soto. (Coord) Políticas Públicas de Educación Superior Intercultural y Experiencias de Diseños Educativos.

www.eumed.net/libros/2010e/830/

http://www.eumed.net/libros/2010e/830/index.htm

Bello Domínguez, Juan. (2010) "El Derecho de los Pueblos Indígenas a una Educación Diferenciada, en el Marco de los Acuerdos Internacionales" en Gunther Dietz, Rafael Regalado Hernández y Ricardo Contreras Soto (Coord) Pluriculturalidad y Educación Tomo III. http://www.eumed.net/ libros/2010d/801/indice.htm

http://www.eumed.net/libros/2010d/801/pgr.jpg

www.eumed.net/libros/2010d/801/

Dietz, G., Et. Al. (2011) Interculturalidad y educación intercultural en México: Un análisis de los discursos nacionales e internacionales en su impacto en los modelos educativos mexicanos. México: Ed. SEP-CGEIB.

Gracia Canclini, N. (1999). La Globalización Imaginada. México: Ed. Paidos

González, T. (2008) Diversidad e Inclusión Educativa: algunas reflexiones sobre el liderazgo en el centro escolar. Madrid, España: Revista Electrónica Iberoamericana sobre Calidad, Eficacia y Cambio en Educación año/vol 6, numero 002.

Habermas J. (1999) La inclusión del otro. Ensayos sobre teoría política. Barcelona: Ed. Paidos.

Sacristán, Gimeno. (2001) Educar y convivir en una cultura global. Las exigencias de la ciudadanía. Madrid: Ed. Morata.

Sartori, G. (2001) La sociedad multiétnica: pluralismo y extranjeros Madrid: Taurus.

Skliar, C., Et. Al. (2008) Conmover la educación. Ensayos para una pedagogía de la diferencia., Buenos Aires, Argentina: Ed. Noveduc

Touraine, A. (1997) ¿'Podremos vivir juntos? México: Ed. FCE

---- (2005) Un Nuevo Paradigma para Comprender el Mundo de Hoy,

España: Ed. Paidos

---- Igualdad y diversidad. (2001) Las nuevas tareas de la democracia. FCE, México. 2001.

UNESCO. (1990) Declaración Mundial sobre Educación para Todos". Satisfacción de las necesidades básicas de aprendizaje. Jomtien, Tailandia. 5 a 9 de marzo.

--- (1994) Declaración de Salamanca, Salamanca España.

--- (2000) Reunión Mundial Educación en Dakar, Senegal.

--- (1997) Nuestra diversidad creativa. Informe de la comisión mundial de cultura y desarrollo. México, ONU/Ediciones UNESCO/Correo de la UNESCO.

\section{JuAN BeLlo Domínguez}

jbello@upn.mx y juanbell@hotmail.com

Doctor en Sociología de la Educación por la UNAM. Miembro del Sistema Nacional de Investigadores, Nivel 1. Profesor Investigador de la Universidad Pedagógica Nacional. Profesor de la Facultad de Estudios Superiores Aragón de la UNAM. Autor y coordinador de libros y artículos especializados sobre Educación, Interculturalidad e Inclusión. 